\title{
Schlafstörungen im Visier
}

Die Häufigkeit von Schlafstörungen spiegeln sich in einem vor allem bei älteren Menschen besorgniserregend hohen Verbrauch an Schlafmitteln. Benzodiazepine können möglicherweise der Entwicklung einer Demenz Vorschub leisten.

Medikamente aus der Klasse der Benzodiazepine sind als Tranquilizer und Schlafmittel hoch wirksam und sicher in der Monotherapie. Doch in Österreich gibt es wahrscheinlich 140.000 Menschen, die in ihrem Leben zumindest eine gewisse Zeit davon abhängig sind, so die Suchthilfe in Wien kürzlich in einer Presseaussendung. Das Problem sei, dass eine einmal aufgetretene Diazepinabhängigkeit nur sehr schwer zu behandeln ist. Eine stationär durchgeführte Entzugstherapie dauert drei bis sechs Wochen. Aufgrund des Suchtpotenzial dieser psychotropen Substanzen fordern viele Experten einen kritischen Einsatz bei strenger Indikationsstellung [1].

\section{Erhöhtes Risiko für Alzheimer-De- menz}

Weiters stehen Benzodiazepine unter Verdacht, dauerhafte kognitive Defizite im Sinne einer Demenz hervorrufen zu können. Sophie Billioti de Gage von der Université de Bordeaux und ihre Kollegen halten es für wahrscheinlich, dass Benzodiazepine mit der Zeit Hirnzellen so schädigen könnten, dass kognitive Hirnleistungen im Alter schneller nachlassen. Die Studie erfasste Daten

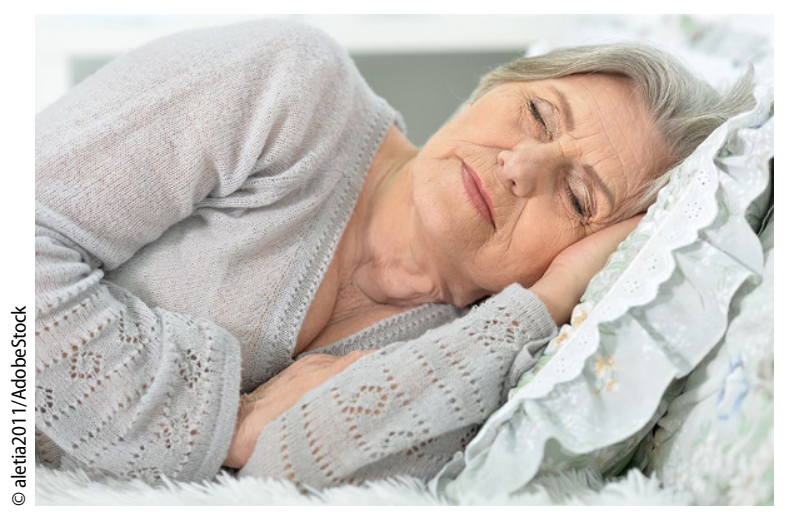

von 1796 über 66-jährigen Menschen, die an Alzheimer erkrankt waren und bereits seit mindestens sechs Jahren an einer kanadischen Langzeitstudie teilnahmen. Als Kontrolle dienten 7184 nach Alter und Geschlecht passend ausgewählte gesunde Personen. Diejenigen, die vor der Demenzdiagnose mindestens drei Monate lang mit einem Benzodiazepin behandelt worden waren, hatten ein um 43 bis $51 \%$ höheres Risiko, an Alzheimer zu erkranken als die anderen. Dieser Zusammenhang war umso stärker, je länger die Medikamenteneinnahme dauerte und je länger die Halbwertszeit, also die Wirkdauer, des jeweiligen Mittels war. Zurzeit sei aber auch eine alternative Erklärung nicht ganz auszuschließen: Eine Alzheimer-Demenz im Frühstadium könnte die Ursache für Angstzustände und Schlafstörungen der Probanden gewesen sein, die deshalb mit Benzodiazepinen behandelt wurden [2]. Auch Zolpidem wird als benzodiazepinähnlich eingestuft [1].

\section{Schlüsselrolle Melatonin}

Mit zunehmendem Alter nimmt die nächtliche Melatoninsekretion ab, was wiederum mit dem gehäuften Auftreten von Schlafstörungen bei älteren Menschen korreliert [3]. Retardiertes Melatonin (Circadin ${ }^{\oplus}$ ) imitiert das natürliche Melatoninprofil durch schrittweise Freisetzung von Melatonin über einen Zeitraum von 8-10 Stunden, was zu erholsamem und natürlichem Schlaf führt. Retardiertes Melatonin ist zugelassen zur Monotherapie für die kurzzeitige Behandlung der primären, durch schlechte Schlafqualität gekennzeichneten Insomnie bei Patienten ab 55 Jahren. Die Tagesdosis von $2 \mathrm{mg}$ retardiertem Melatonin wird eine bis zwei Stunden vor dem Schlafengehen und nach der letzten Mahlzeit eingenommen. Die Einnahme kann bis zu 13 Wochen lang fortgesetzt werden [4]. Betroffene gewinnen somit oftmals eine gute Schlafqualität und in der Folge eine verbesserte Tagesfunktionalität ohne die morgendlichen Nachwirkungen, die nach der Einnahme älterer Hypnotika auftreten können. Denn neben der Möglichkeit einer längerfristigen Therapie, stehen die Erhaltung der Schlafqualität, der erholsame Schlaf und die Morgendliche Aufmerksamkeit an oberster Stelle der Zielkriterien für optimale Schlafmittel [5].

\section{Literatur}

1. Broschüre „Empfehlung zur ärztlichen Verordnung von Benzodiazepinen. 2017, Suchthilfe Wien

2. Billioti de Gage S (2014) Benzodiazepine use and risk of Alzheimer's disease: case-control study. BMJ 349:g5205. https://dx.doi.org/10.1136/bmj.g5205

3. Leger D, Laudon M, Zisapel N (2004) Nocturnal 6-sulfatoxymelatonin excretion in insomnia and is relation to the response to melatonin replacement therapy. Am J Med 116(2):91-95

4. Fachinformation Circadin ${ }^{\oplus} 2$ mg Retardtabletten, Zugegriffen: Juli 2016

5. http://www.ema.europa.eu/docs/en_GB/ document_library/Scientific_guideline/2011/02/WC500102351.pdf; Zugegriffen: 17.11.2011

psychopraxis. neuropraxis $2017 \cdot 20: 194$ https://doi.org/10.1007/s00739-

017-0433-3

(c) Springer-Verlag GmbH Austria 2017 